\title{
Erratum to: A Singular Differential Equation Stemming from an Optimal Control Problem in Financial Economics
}

\author{
Pavol Brunovský1 ${ }^{1}$ - Aleš Černý ${ }^{2}$. \\ Michael Winkler ${ }^{3}$
}

Published online: 28 December 2016

(C) Springer Science+Business Media New York 2016

\section{Erratum to: Appl Math Optim (2013) 68:255-274 DOI 10.1007/s00245-013-9205-5}

The sentence at the top of page 261 contains an error in the expression for $b$. The text should read, "From here it is readily seen that the radius of convergence of the power series (2.9) is nil when $a<\frac{3}{2}$ and $b \in\left(\frac{3}{4}-\frac{3}{2} a, 2-2 a\right), \ldots$. We are grateful to Pavol Quittner for bringing this typo to our attention. A tighter estimate of the zero-radius region and a deeper analysis of the asymptotic properties of solutions is provided in [1].

\section{Reference}

1. Quittner, P.: Higher order asymptotics of solutions of a singular ODE. Asymptot. Anal. 94(3-4), 293-308 (2015)

The online version of the original article can be found under doi:10.1007/s00245-013-9205-5.

Pavol Brunovský

brunovsky@fmph.uniba.sk

Aleš Černý

ales.cerny.1@city.ac.uk

Michael Winkler

michael.winkler@uni-due.de

1 Department of Applied Mathematics and Statistics, Comenius University Bratislava, 84248 Bratislava, Slovakia

2 Cass Business School, City University London, 106 Bunhill Row, London EC1Y 8TZ, UK

3 Institut für Mathematik, Universität Paderborn, 33098 Paderborn, Germany 